\title{
EXPANDING AXIAL WAVE ON A SUBMERGED CYLINDRICAL SHELL*
}

\author{
BY \\ TSUN C. FANG \\ Grumman Aerospace Corp., Bethpage, New York \\ AND \\ JEROME M. KLOSNER \\ Polytechnic Institute of Brooklyn
}

\begin{abstract}
A double transform method is used to determine the response of a submerged, infinitely long, circular cylindrical shell to a plane acoustic wave which acts initially at an isolated cross section, and then proceeds to propagate along the axis of the cylinder, symmetrically with respect to that cross section.

The application of the saddle point method to the inversion integral results in an asymptotic solution, valid for long time. Comparisons are made with the analogous steady-state solution obtained by using piston theory to describe the effects of the fluid on the shell.

I. Introduction. The investigation of the interaction of acoustic media and shell structures has been extensively treated in the literature. One class of these studies has concerned itself with the response of structures to engulfing pressure waves. In the vast majority of these studies, the reflected acoustic field is accounted for by making a plane wave assumption, that is, by using the so-called piston theory [1], [2]. This simplification results in the uncoupling of the acoustic and elastic fields, thereby significantly reducing the analytical difficulties. It is well known that the use of piston theory, which for short time responses is reasonably valid, is highly suspect for long times. However, since solutions of the exact coupled fields were nonexistent until recently, it was impossible to determine the range of applicability of these approximate solutions. Haywood [3] investigated a simplified form of the plane problem of the engulfing wave by assuming that the shell is suddenly subjected to a hydrostatic pressure. Neglecting the mass of the shell, and using a cylindrical wave approximation, he showed that the plane wave approximation underestimated the shell response.

Nore recently several papers have appeared in which the exact solutions of some interaction problems have been obtained and then compared to the approximate solutions. In the first of these, Herman and Klosner [4] determined the response of a circular cylindrical shell to a sudden pressure increase in its surrounding fluid. In order to simplify the wathemitical lificulties, and yet retain the characteristic form of the time dependency, the pressure was assumed to vily periodically along the axis of the shell. The results in-

* lieceived July 17, 1969; revicel version received November 1, 1969. The results presented in this paper are hased on a Ph.1). disortation by the first author submitted to the Polytechnic Institute of Brooklyn, June 1969. The work was sponsored by the Office of Naval Research, Department of the Navy, under Contract No. Nonr 839(40)FBM, Project No.NR 064-427.
\end{abstract}


dicated that for very long and short wavelengths, the approximate solutions yielded results with similar characteristics to those obtained from the exact solution. However for the intermediate range, the approximations were entirely inadequate. An explanation of this phenomenon is suggested by noting that the exict solution contained both radiating and nonradiating components, while the pline and cylindrical wave approximations consisted only of radiating modes. Thus, when a significant nonradiating component is present, the form of the response function differs ridically from that predicted by the approximations. Berglund and Klosner [5] extended this study with an investigation of a more realistic situation. They considered the transient dynamic response of a periodically ring-reinforced, infinitely long, circular cylindrical shell to a uniform pressure suddenly applied through the surrounding accoustic medium. It was shown, for the rigid-ring case, that the cylindrical wave approximation was adequate, while this was not so for the flexible ring configuration. In the latter structure, significant nonradiating or undamped components of the shell response were present, and thus the approximations had to be applied with care.

The present study is a continuation of a general investigation which has been undertaken at the Polytechnic Institute of Brooklyn to determine the dynamic response of submerged structures, and in doing so, to assess also the applicability of piston theory or other approximations. The origin of the problem stems from an investigation by Carrier [6] of the steady-state response of a submerged, infinitely long, circular cylindrical shell subjected to an axially progressing acoustic wave. He obtained a solution by making the fundamental assumption of piston theory. In an attempt to re-investigate the same problem by using the exact, line:arized acoustic field equations, it was found that no physically meaningful steady-state solution exists; and that, if one wishes to use the exact acoustic equations, Carrier's problem is not well posed. It was, therefore, in order to overcome this deficiency and yet maintain the physical structure of Carrier's problem, that the present investigation was undertaken.

Consider a submerged, infinitely long, circular cylindrical shell, initially at rest, subjected to a plane acoustic wave which initially acts at a single cross section and propagates along the axis of the cylinder symmetrically with respect to that cross section. Certainly for long time the physical nature of the problem resembles the Carrier problem, and thus it is appropriate to compare the asymptotic solution, valid for long time, to that obtained from the Carrier solution in which piston theory wits applied.

It should be noted thiat in a recent study IIermamn and Russell [7] attempted to reinvestigate the Carrier problem and came to the conclusion that no physically meaningful steady-state solution exists. To simplify the problem they considered instead the response of a submerged plate on an elatstic foundation and assumed that the moving load progresses with supersonic or subsonic velocities. They showed that piston theory is applicable only in cases where the loads move with velocities very much greater than the sonic velocity of the fluid and questioned the existence of a steady-state solution for sonic velocities.

II. Governing field equations. (insiler an infinitely long, circular cylindrical shell with uniforin thickness $h$ and median surface radius $a$. The submerged shell is subjected to a plane acrustic wave which initinlly acts on the cross section $x=0$, and propagates symmetrically with respect to that cross section along the cylinder axis (Fig. 1). The incident pressure is assumed to be $p, I(\sigma)$, where $\sigma=\tau \mp x$ (the upper sign is for positive $\dot{x}$ and the lowr sign for negative $x) ; I(\sigma)$ is the Heaviside step function.

Neglecting the axial inertiat term and setting the longitudinal stress resultant $N_{x}=0$, 
we may express the uncoupled Timoshenko (or Donnell) shell equation as

$$
w_{, x x x x}+4 K^{4} w+4 K^{4}\left(c / c_{s}\right)^{2} w_{, \tau \tau}=4 K^{4}(a / h)(p / E) .
$$

The axisymmetric form of the acoustic field equation is

$$
\varphi_{, r r}+(1 / r) \varphi_{, r}+\varphi_{, x x}=\varphi_{. \tau \tau}
$$

and thus the radiated velocity and pressure can be written as

$$
\begin{gathered}
\left(V_{r}\right)_{i}=-(1 / a) \varphi_{, i} \quad(j=r, x), \\
p_{r}=(\rho c / a) \varphi_{, \tau} .
\end{gathered}
$$

Initially, the radiated velocity and pressure of the acoustic medium as well as the velocity and displacement of the shell vanish; thus

$$
\begin{gathered}
\varphi(r, x, 0)=\varphi_{, \tau}(r, x, 0)=0, \\
w(x, 0)=w_{, \tau}(x, 0)=0 .
\end{gathered}
$$

At the shell-fluid interface $(r=1)$, we have the boundary condition

$$
w_{, \tau}(x, \tau)=(1 / a c) \varphi_{, r}(1, x, \tau) .
$$

The total pressure, $p$, acting on the shell is the resultant of the pressure exerted by the incoming and radiated acoustic waves, i.e.,

$$
p=p_{0} H(\sigma)+(\rho c / a) \varphi_{, \tau}(1, x, \tau) .
$$

III. Transformed solution. Upon substituting Eq. (8) into Eq. (1) and depressing $x$ and $\tau$ by applying the Fourier and Laplace transform to the resulting expression and Eq. (2), we obtain

$$
\Omega^{4} \bar{W}+4 K^{4} \bar{V}\left[1+s^{2}\left(\frac{c}{c_{s}}\right)^{2}\right]=4 K^{-4} \frac{a}{h} \frac{p_{0}}{E^{\prime}}\left(2 / k^{2}\right)+4 K^{4}\left(\frac{a}{h}\right) \frac{\rho c}{a \bar{E}} s \bar{\Phi} \quad(1, \Omega, s)
$$

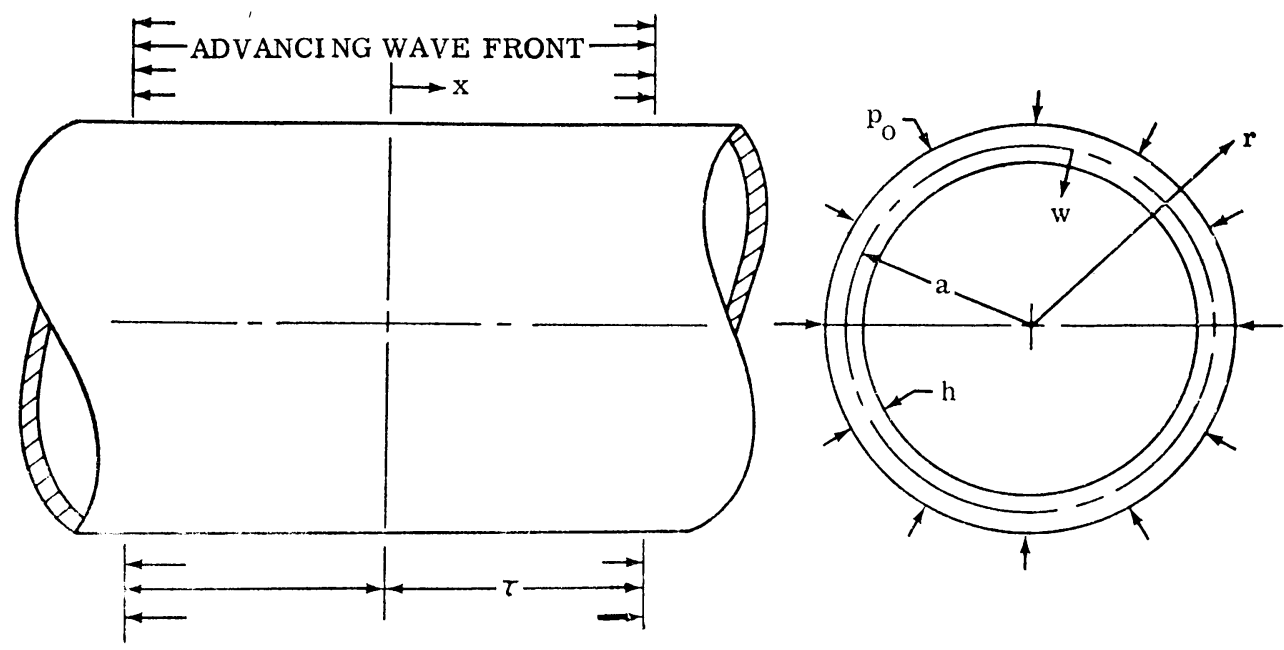

FIG. 1. Shell geometry 
and

$$
\Phi_{, r r}+(1 / r) \Phi_{, r}-k^{2} \bar{\Phi}=0 ; \quad k^{2}=\Omega^{2}+s^{2}
$$

where $W(r, \Omega, \tau)$ and $\bar{W}(r, \Omega, s)$ are the Fourier and Laplace transforms of $v(r, x, \tau)$.

Taking account of the interface condition (7), we may express the outgoing wave solution of Eq. (10) as

$$
\bar{\Phi}=-\left(a c s \mid \bar{V} / k K_{1}(k)\right) K_{0}(k r)
$$

Substituting Eq. (11) into Eq. (9), and letting $c_{s}=\left(E / \rho_{s}\right)^{1 / 2}$, we obtain the transform of the deflection function,

$$
\bar{W}(\Omega, s)=\frac{8 K^{4} \frac{a}{h} \frac{p_{0}}{E}}{k^{2}\left\{\Omega^{4}+4 K^{4}+4 K^{4} s^{2}\left(\frac{c}{c_{s}}\right)^{2}\left[1+\left(\frac{\rho}{\rho_{s}}\right)\left(\frac{a}{h}\right) \frac{K_{0}(k)}{k K_{1}(k)}\right]\right\}} .
$$

IV. Inversion of Laplace transform. The Laplace inversion integral can be evaluated by use of the Cauchy residue theorem. In so doing, the path of integration is closed on the left half of the complex $s$-plane. Since $k$ is a multiple valued function with branch points at $s= \pm i \Omega$, the path of integration is as shown in Fig. 2. By Jordan's Lemma, the integral along the semicircle $C_{r}$ vanishes and thus

$$
\begin{aligned}
W(\Omega, \tau)=\int_{\beta-i \infty}^{\beta+i \infty} e^{\tau s} \bar{W}(\Omega, s) d s & =2 \pi i \sum_{a}(\operatorname{Res})_{a} \\
& -\int_{L_{1}+C_{1}+L_{2}} e^{\tau s} \bar{V}(\Omega, s) d s-\int_{L_{3}+C_{2}+L_{4}} e^{\tau s} \bar{W}(\Omega, s) d s .
\end{aligned}
$$

The poles are located by setting the bracketed portion of the denominator of Eq. (12) equal to zero:

$$
\Omega^{4}+4 K^{4}+4 K^{4} s^{2}\left(\frac{c}{c_{s}}\right)^{2}\left[1+\left(\frac{\rho}{\rho_{s}}\right)\left(\frac{a}{h}\right) \frac{K_{0}\left(k_{i}\right)}{k K_{1}(k)}\right]=0 .
$$

Suppose $s=s_{a},(q=1,2, \cdots)$, are the roots of Eq. (14). Then the position of the poles depends on the values of $\Omega$ and thus defines a function $s_{q}=s_{q}(\Omega)$. There are an infinite number of such functions. For $\Omega$ real, $\Omega=\Omega_{0}$, there are complex, imaginary, and real values of the poles $s_{4}$. However, the real and complex roots are unimportant for large values of $\tau$, since they have neg: tive real parts, and thus we are concerned only with the imaginary roots. A question then arises as to whether to include the residues of all of the poles occurring on the imaginary axis. This can be answered by anticipating the inverse Fourier transformation of the space variable, in which the integration is to be performed limitingly close to, but just above, the real axi: in the $\Omega$-plane. Thus the $\Omega$ to be used in calculating the poles $s_{a}$ should have a vanishingly small but positive imaginary component $i \alpha$; accordingly,

$$
s_{u}(\Omega)=s_{4}\left(\Omega_{11}+i \alpha\right)=s_{4}\left(\Omega_{1}\right)+\left(d s_{q} / d \Omega\right)_{\Omega=\Omega, i} i \alpha .
$$

Thus we see that when $s_{q}\left(\Omega_{0}\right)$ is imaginary, $s_{q}$ will have a small real part whose sign is negative if and only if $\operatorname{Im}\left(d s_{q} / d \Omega\right)=\beta, \beta>0$. Therefore, in obtaining the residues of the poles in the left-hand s-plane, we include only those poles for which $\operatorname{Re}\left(i d s_{a} / d \Omega\right)<0$. 
We now let

$$
s_{a}=i t_{a} ; \quad k_{a}^{2}=\Omega^{2}-t_{a}^{2} .
$$

Hence from Eq. (12) we obtain the following expression for the residues of the simple poles:

$$
\operatorname{Res}_{u}=\left(a p_{0} / i h E\right) F(\Omega) e^{i=t_{u}(\Omega)}
$$

where

$$
F(\Omega)=t_{a}\left[l_{i}^{2}\left(\frac{\Omega^{4}}{4 K^{-j}}+1\right)+\frac{1}{2} t_{1}^{4}\left(\frac{c}{c_{s}}\right)^{2}\left(\frac{\rho}{\rho_{s}}\right)\left(\frac{a}{h}\right)\left(1-\frac{K_{0}^{2}\left(k_{q}\right)}{K_{1}^{2}\left(k_{q}\right)}\right)\right]^{-1} .
$$

Let us now consider the branch cut integrals $B$, where

$$
B=\int_{l} \bar{W}(\Omega, s) r^{r_{s}} d s=\int_{1} \frac{2 \frac{a}{h} \frac{p_{0}}{k^{2}} e^{r s} d s}{k^{2}\left\{\frac{\Omega^{4}}{4 K^{4}}+1+s^{2}\left(\frac{c}{c_{s}}\right)^{2}\left[1+\left(\frac{\rho}{\rho_{s}}\right)\left(\frac{a}{h}\right) \frac{K_{0}(k)}{k K_{1}(k)}\right]\right\}}
$$

and $l=L_{1}+C_{1}+L_{2}+L_{3}+C_{2}+L_{4}$.

If the radii of $C_{1}$ and $C_{2}$ are chosen to be $\epsilon(\ll 1)$, then on the path $C_{1}$

$$
\begin{aligned}
s & =i \Omega+\epsilon e^{i \theta} \approx i \Omega ; \quad s^{2} \approx-\Omega^{2} ; \\
k_{+}^{2} & =\Omega^{2}+s^{2} \approx 2 i \epsilon \Omega^{i \theta}
\end{aligned}
$$

and the integral about the path $C_{3}$ may thus be expressed as

$$
I_{c_{1}}=\int_{C_{1}} \bar{W}(\Omega, s) e^{\tau_{t}} d s=\frac{a p_{0}}{h \bar{E}^{\prime}} e^{e^{i \Omega_{\tau}}} \int_{x}^{-\pi} \frac{d \theta}{\frac{\Omega^{4}}{4 K^{4}}+1-\Omega^{2}\left(\frac{c}{c_{s}}\right)^{2}\left[1+\left(\frac{\rho}{\rho_{s}}\right)\left(\frac{a}{h}\right) \frac{K_{0}\left(k_{+}\right)}{k_{+} K_{1}\left(k_{+}\right)}\right]} .
$$

The integral about $C_{2}, I_{c_{2}}$, is identical to the above except that $\Omega$ must be replaced by its negative value and thus $k_{+}$by $k_{-}\left(k_{-}^{2} \approx-2 i \epsilon \Omega e^{i \theta}\right)$. As $|k| \rightarrow 0, K_{0}(k) /\left(k K_{1}(k)\right) \approx$ $\ln k \rightarrow-\infty$ and thus $I_{C_{1}}$ and $I_{C_{2}}$ vanish when $\Omega \neq 0$. However, the contribution from $I_{C_{1}}$ and $I_{C_{2}}$ at $\Omega=0$ is singular and their evaluation will be pursued in detail later.

We consider next the contribution from the paths $L_{1}, L_{2}, L_{3}$ and $L_{4}$. From Fig. 2, we note that : (i) along $L_{1}, s=s_{1}=i \Omega+\rho e^{i \pi}=i \Omega-\rho$, (ii) along $L_{2}, s=s_{2}=i \Omega+$ $\rho e^{-i \pi}=i \Omega-\rho$, (iii) along $L_{3}, s=s_{3}=-i \Omega+\rho e^{i \pi}=-i \Omega-\rho$, (iv) along $L_{4}, s=s_{4}=$ $-i \Omega+\rho e^{-i \pi}=-i \Omega-\rho$. Hence

$$
s_{1}^{2}=s_{2}^{2}=\rho^{2}-\Omega^{2}-2 i \rho \Omega ; \quad s_{3}^{2}=s_{1}^{2}=\rho^{2}-\Omega^{2}+2 i \rho \Omega
$$

and from the second of Egs. (10), we find that

$k_{1}=k e^{(i \theta / 2)+\pi i} ; \quad k_{2}=k e^{i \theta / 2}=-k_{1} ; \quad k_{3}=k e^{-i \theta / 2} ; \quad k_{4}=k e^{(-i \theta / 2)-\pi i}=-k_{3}$

where

$$
k=\rho\left(1+4 \Omega^{2} / \rho^{2}\right)^{1 / 4} ; \quad \theta=-\tan ^{-1}(2 \Omega / \rho) .
$$




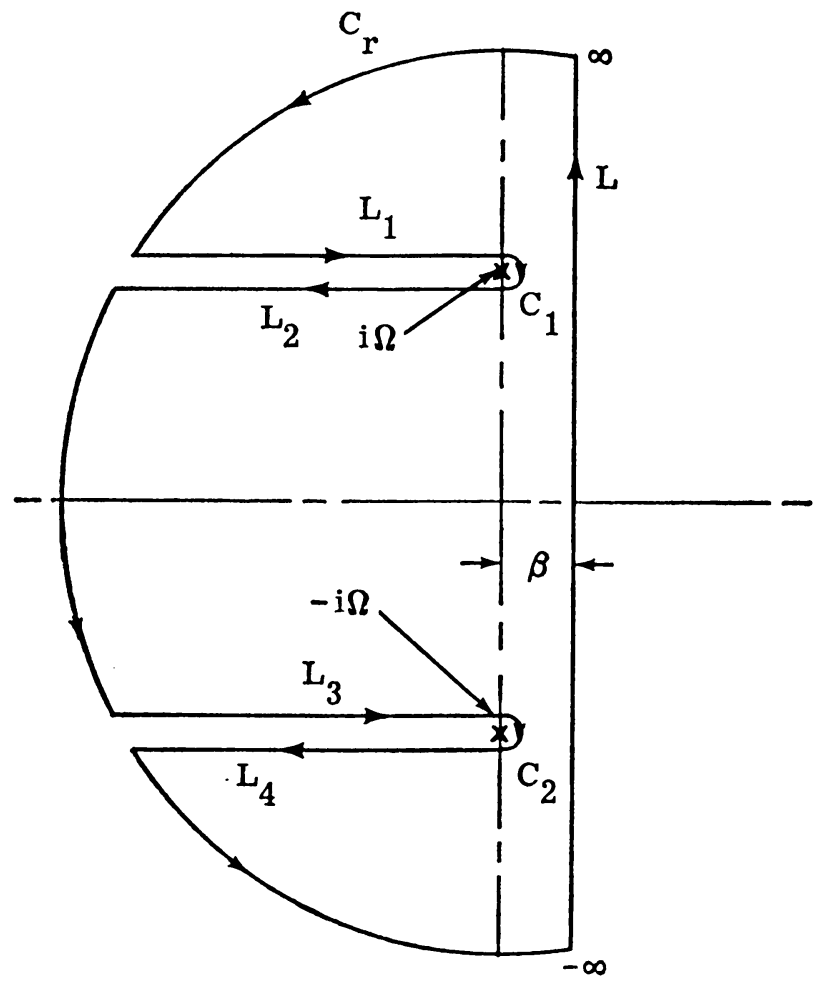

FIc. 2. Contour in s-plane.

Thus

$$
\begin{aligned}
\int_{L_{n}} \bar{W}(\Omega, s) e^{\tau s} d s=(-1)^{n} 2 \frac{a p_{10}}{h E} e^{i \Omega \tau} \\
\quad \cdot \int_{0}^{\infty} \frac{e^{-\rho \tau} d \rho}{k_{n}^{2}\left\{\frac{\Omega^{4}}{4 K^{4}}+1+s_{n}^{2}\left(\frac{c}{c_{s}}\right)^{2}\left[1+\left(\frac{\rho}{\rho_{s}}\right)\left(\frac{a}{h}\right) \frac{K_{0}\left(k_{n}\right)}{k_{n} K_{1}\left(k_{n}\right)}\right]\right\}} \quad(n=1,2,3,4) .
\end{aligned}
$$

Taking note of Eqs. (21), (22) and Eq. (24), we have

$$
\int_{L_{1}+L_{3}} \bar{V}(\Omega, s) e^{\tau s} d s=2\left(\frac{\rho}{\rho_{s}}\right)\left(\frac{a}{h}\right)^{2}\left(\frac{c}{c_{s}}\right)^{2} \frac{p_{0}}{E} e^{i \Omega l r} \quad\left(M_{1}+N_{1}\right)
$$

and

$$
\int_{L_{3}+L .} \bar{W}(\Omega, s) e^{\tau s} d s=2\left(\frac{\rho}{\rho_{s}}\right)\left(\frac{a}{h}\right)^{2}\left(\frac{c}{c_{s}}\right)^{2} \frac{p_{0}}{E^{i}} e^{-i S l \tau} \quad\left(M_{3}+N_{3}\right),
$$

where

$$
M_{i}=-\int_{0}^{\delta} \frac{s_{j}^{2}}{k_{j}} H_{i}(\rho) B_{j}(\rho) e^{-\rho \tau} d \rho ; \quad N_{i}=-\int_{\delta}^{\infty}{\stackrel{s}{k_{j}}}_{k_{j}} I_{j}(\rho) B_{i}(\rho) e^{-\rho \tau} d \rho \quad(j=1,3),
$$

and

$$
H_{i}(\rho)=\left[g\left(k_{i}\right) g\left(-k_{j}\right)\left\{k_{i} K_{1}\left(k_{i}\right) i i-k_{j} K_{1}\left(-k_{i}\right)\right\}\right]^{-1},
$$




$$
\begin{aligned}
& g\left(k_{i}\right)=\frac{\Omega^{4}}{4 K^{4}}+1+s_{i}^{2}\left(\frac{c}{c_{s}}\right)^{2}\left[1+\left(\frac{\rho}{\rho_{s}}\right)\left(\frac{a}{h}\right) \frac{K_{0}\left(k_{j}\right)}{k_{i} K_{1}\left(k_{i}\right)}\right], \\
& B_{i}(\rho)=K_{1}\left(-k_{j}\right) K_{0}\left(k_{i}\right)+K_{1}\left(k_{j}\right) K_{0}\left(-k_{j}\right)=\pi i / k_{i} .
\end{aligned}
$$

The value of $\delta$ is chosen so that the asymptotic form of the integrand is sufficiently accurate for values of $\rho \geq \delta$.

Evaluation of branch cut integrals. It can easily be shown that

where

$$
M I_{i} \approx-\pi i P_{i}\left(\bar{\delta}_{1}\right)\left(e^{-\delta_{1} \tau} / \tau\right)
$$

$$
P_{j}\left(\bar{\delta}_{1}\right)=\left.\left(s_{i}^{2} / h_{j}^{2}\right) H_{j}(\rho)\right|_{\rho=\bar{\delta}_{1}} ; \quad \delta_{1} \leq \bar{\delta}_{1} \leq \delta ; \quad \delta_{1} \ll 1 .
$$

Also upon using the asymptotic forms of the Bessel functions, we have

$$
N_{i} \sim \int_{\delta}^{\infty} Q_{j}(\rho) e^{-\rho \tau} d \rho=Q_{i}(\bar{\delta})\left(e^{-\delta \tau} / \tau\right)
$$

where

$$
\left.Q_{j}(\bar{\delta}) \sim \frac{s_{i}^{2}\left(2+\frac{15}{32 k_{j}^{2}}+\cdots\right)}{k_{i}^{3} g\left(k_{j}\right) g\left(-k_{j}\right)}\right|_{\rho=\delta_{1}} ; \quad \delta \leq \bar{\delta} \leq \infty .
$$

Finally, we substitute Eqs. (30), (32) into Eq. (25) and then into Eq. (19), thereby obtaining the following expressions when $\Omega \neq 0$ :

$$
\begin{aligned}
B=\int_{l} \bar{W}(\Omega, s) e^{\tau s} d s \approx 2\left(\frac{\rho}{\rho_{s}}\right) & \left(\frac{a}{h}\right)^{2}\left(\frac{c}{c_{s}}\right)^{2} \frac{p_{0}}{E}\left[\left(-\pi i P_{1}\left(\bar{\delta}_{1}\right) \frac{e^{-\delta_{1} \tau}}{\tau}+Q_{1}(\bar{\delta}) \frac{e^{-\delta \tau}}{\tau}\right) e^{i \Omega \tau}\right. \\
& \left.+\left(-\pi i P_{3}\left(\bar{\delta}_{1}\right) \frac{e^{-\delta_{1} \tau}}{\tau}+Q_{3}(\bar{\delta}) \frac{e^{-\delta \tau}}{\tau}\right) e^{-i \Omega \tau}\right] \quad(\Omega \neq 0) .
\end{aligned}
$$

For $\Omega=0$ we obtain a similar expression with $e^{i \Omega \tau}$ and $e^{-i \Omega r}$ replaced by 1 , and thus the branch point contributions mily be expressed as

$$
B=O\left(e^{-\delta \tau} / \tau\right)+O\left(e^{-\delta_{1} \tau} / \tau\right)+I_{C_{1}}+I_{C_{2}} \quad(\Omega=0) .
$$

Since we seek an asymptotic solution for liarge positive real values of $\tau$, and $\delta$ and $\delta_{1}$ are positive real, then the exponential terms of Eqs. (34) and (35) are rather small and negligible. Hence Eq. (13) reduces to

$$
W(\Omega, \tau)=\int_{\beta-i \infty}^{\beta+i \infty} e^{\tau s} \bar{W}(\Omega, s) d s=2 \pi i \sum_{a}(\operatorname{Res})_{a}-I_{C_{2}}-I_{C_{2}}
$$

or

$$
\begin{aligned}
W(\Omega, \tau)=\frac{a p_{0}}{i h E} \sum_{a} F(\Omega) e^{i \tau t_{0}(\Omega)} \\
\quad-\frac{a p_{0}}{2 \pi i h E} \frac{e^{i \Omega r}}{\Omega} \int_{\pi}^{-\pi} \frac{d \theta}{\frac{\Omega^{4}}{4 K^{4}}+1-\Omega^{2}\left(\frac{c}{c_{s}}\right)^{2}\left[1+\left(\frac{\rho}{\rho_{s}}\right)\left(\frac{a}{h}\right) \frac{K_{0}\left(k_{+}\right)}{k_{+} K_{1}\left(k_{+}\right)}\right]} \\
+\frac{a p_{0}}{2 \pi i h L} \frac{e^{-i: l r}}{\Omega} \int_{\pi}^{-\pi} \frac{d \theta}{\frac{\Omega^{4}}{4 K^{4}}+1-\Omega^{2}\left(\frac{c}{c_{s}}\right)^{2}\left[1-\left(\frac{\rho}{\rho_{s}}\right)\left(\frac{a}{h}\right) \frac{K_{0}\left(k_{-}\right)}{k_{-} K_{1}\left(k_{-}\right)}\right]} .
\end{aligned}
$$


For clarity, it will be repeated that the above two integrals representing $I_{C_{2}}$ and $I_{C}$, vanish except for $\Omega=0$.

V. Inversion of Fourier transform. The substitution of Eq. (37) into the Fourier inversion integral then yields the expression for the radial shell deflection

$$
\begin{aligned}
& \frac{w(x, \tau)}{\frac{a}{h} \frac{p_{0}}{E}}=\frac{1}{2 \pi i} \sum_{a} I_{a} \\
& \quad-\frac{1}{4 \pi^{2}} i \int_{-\infty+i \alpha}^{\infty+i \alpha} \frac{e^{i \Omega(\tau-x)}}{\Omega} d \Omega \int_{\pi}^{-\pi} \frac{d \theta}{\frac{\Omega^{4}}{4 K^{4}}+1-\Omega^{2}\left(\frac{c}{c_{s}}\right)^{2}\left[1+\left(\frac{\rho}{\rho_{s}}\right)\left(\frac{a}{h}\right) \frac{K_{0}\left(k_{+}\right)}{k_{+} K_{1}\left(k_{+}\right)}\right]} \\
& \quad+\frac{1}{4 \pi^{2}} i \int_{-\infty+i \alpha}^{\infty+i \alpha} \frac{e^{-i \Omega(\tau+x)}}{\Omega} d \Omega \int_{\pi}^{-\pi} \frac{d \theta}{\frac{\Omega^{4}}{4 K^{4}}+1-\Omega^{2}\left(\frac{c}{c_{s}}\right)^{2}\left[1+\left(\frac{\rho}{\rho_{s}}\right)\left(\frac{a}{h}\right) \frac{K_{0}\left(k_{-}\right)}{k_{-} K_{1}\left(k_{-}\right)}\right]}
\end{aligned}
$$

where the $q$ th term of the above series is

$$
I_{a}=\int_{-\infty+i \alpha}^{\infty+i \alpha} F(\Omega) e^{\tau(\Omega)} d \Omega ; \quad h(\Omega)=i\left[t_{q}(\Omega)-(x / \tau) \Omega\right] .
$$

The complexity of both $F(\Omega)$ and $t_{q}(\Omega)$ makes it impossible, in general, to evaluate the first integral by simple means. However, provided $\tau$ is large, real and positive, the method of steepest descent [8]-[11] can be used to obtain an asymptotic approximation to the integral. The second and third integrals can be evaluated by use of the residue theorem and present no difficulties. Thus, we center our attention on the asymptotic evaluation of the first integral.

Determination of saddle point. The saddle point, $\bar{\Omega}$, associated with the $t_{a}$ root is obtained by determining the value of $\Omega$ for which $h^{\prime}(\Omega)=0$; i.e.,

$$
h^{\prime}(\bar{\Omega})=i\left(\frac{d t_{a}}{d \Omega}-\frac{x}{\tau}\right)_{\Omega=\bar{\Omega}}=0
$$

and thus

$$
\left.\frac{d t_{a}}{d \Omega}\right|_{\Omega=\bar{\Omega}}=\frac{d t_{a}}{d \bar{\Omega}}=\frac{x}{\tau} .
$$

Substituting Eq. (16) into Eq. (14) and differentiating and then appealing to Eq. (41), we obtain

$$
\frac{2 \bar{\Omega}_{a}\left\{\bar{k}_{a}^{2} \bar{\Omega}^{2}+K^{4} \bar{t}_{a}^{2}\left(\frac{c}{c_{s}}\right)^{2}\left(\frac{\rho}{\rho_{s}}\right)\left(\frac{a}{h}\right)\left[1-\left(\frac{K_{0}\left(\bar{k}_{q}\right)}{K_{1}\left(\bar{k}_{q}\right)}\right)^{2}\right]\right\}}{\bar{k}_{a}^{2}\left(\bar{\Omega}^{4}+4 K^{4}\right)+2 K^{4} t_{a}^{4}\left(\frac{c}{c_{s}}\right)^{2}\left(\frac{\rho}{\rho_{s}}\right)\left(\frac{a}{h}\right)\left[1-\left(\frac{K_{0}\left(\bar{k}_{q}\right)}{K_{1}\left(\bar{k}_{q}\right)}\right)^{2}\right]}=\frac{x}{\tau}
$$

where

$$
\bar{k}_{a}=\left(\bar{\Omega}^{2}-\bar{t}_{a}^{2}\right)^{1 / 2} \text {. }
$$

Of course $\bar{t}_{a}(\bar{\Omega})$ must satisfy Eq. (14), namely

$$
\bar{t}_{a}^{2}=\bar{\gamma}\left[1 \pm\left(1-\left(\bar{k}_{a}^{4}+4 K^{4}\right) / \bar{\gamma}^{2}\right)^{1 / 2}\right]
$$


where

$$
\bar{\gamma}=\gamma\left(\bar{k}_{q}\right)=2 K^{4}\left(\frac{c}{c_{s}}\right)^{2}\left[1+\left(\frac{\rho}{\rho_{s}}\right)\left(\frac{a}{h}\right) \frac{K_{0}\left(\bar{k}_{q}\right)}{\overline{k_{q}} K_{1}\left(\overline{k_{q}}\right)}\right]-\bar{k}_{a}^{2} .
$$

In Eq. (41), $\tau$ locates the wave front and $x$ is the axial coordinate of the shell at which the radial deflection is to be evaluated. Both $\tau$ and $x$ are given real quantities and thus in order to satisfy Eq. (42), $\bar{\Omega}, \bar{t}_{a}, \bar{k}_{q}$ must all be real. For given $x$ and $\tau$, Eqs. (42)-(44) yield three simultaneous equations for the three unknowns, $\bar{\Omega}, \bar{t}_{q}$ and $\bar{k}_{q}$. Since Eqs. (43) and (44) are even functions of both $\bar{\Omega}$ and $\bar{t}_{a}$, the saddle points occur in pairs, namely at $\pm \bar{\Omega}$. As $\bar{\Omega}, \bar{t}_{a}$, and $\bar{k}_{q}$ are real and $K_{0}\left(\bar{k}_{q}\right)<K_{1}\left(\bar{k}_{q}\right)$, then by observing Eq. (42), we have

$$
\operatorname{sgn}(x / \tau)=\operatorname{sgn}\left(\bar{\Omega} \bar{t}_{a}\right) .
$$

Use of this relationship will be made later.

The asymptotic evaluation of Eq. (39) can be carried out by deforming the original path of integration to a contour $C^{\prime}=C_{1}^{\prime}+C_{2}^{\prime}$, which passes through the saddle points in the directions of steepest descent (see Fig. 3 and Appendix A). The principal contributions to the integral then come from the parts of the paths near the saddle points and from any poles that are included between the region enclosed by the original and deformed contours.

Saddle point of order 1: second order solution. We consider a saddle point of order 1, i.e., one for which the $h^{\prime \prime}(\bar{\Omega}) \neq 0$. In the immediate vicinity of the saddle point $\bar{\Omega}$, we expand both $h(\Omega)$ and $F(\Omega)$ into Taylor series. Approximating $h(\Omega)$ by terms up to and including its second derivatives thus yields the second order approximation of the integral of Eq. (39) evaluated along the deformed path

$$
\begin{aligned}
I_{a}^{\prime}=\int_{C^{\prime}} F(\Omega) \exp \left[i \tau\left(t_{q}(\Omega)-\frac{x}{\tau} \Omega\right)\right] d \Omega \\
=e^{\tau h(\bar{\Omega})} \sum_{j=0}^{\infty} \frac{F^{(i)}(\bar{\Omega})}{j !} \int_{C^{\prime}}(\Omega-\bar{\Omega})^{i} \exp \left(\frac{\tau h^{\prime \prime}(\bar{\Omega})}{2 !}(\Omega-\bar{\Omega})^{2}\right) d \Omega
\end{aligned}
$$

where $C^{\prime}$ is the deformed path of integration and consists of $C_{1}^{\prime}$, passing through the saddle point $+\bar{\Omega}$, and $C_{2}^{\prime}$, which passes through $-\bar{\Omega}$. It can be shown after some intermediate algebra [12] that the contribution to the integral of Eq. (39) from the path of steepest descent $C_{1}^{\prime}$ is

$$
\frac{\left.I_{a}^{\prime}\right|_{\bar{\Omega}}}{2 \pi i}=\left(\frac{2}{\pi \tau\left|d^{2} \bar{t}_{a} / d \bar{\Omega}\right|^{2}}\right)^{1 / 2}\left(\frac{e^{i \psi}}{2 i}\right)\left(F(\bar{\Omega})+\sum_{i=1}^{\infty} \frac{1.3 \cdots(2 j-1)}{(2 j) !} \frac{i^{i} F^{(2 j)}(\bar{\Omega})}{\left(\tau d^{2} t_{a} / d \bar{\Omega}^{2}\right)^{i}}\right)
$$

where

$$
\begin{aligned}
& \psi=\tau \bar{t}_{a}-x \bar{\Omega}+(\pi / 4) \operatorname{sgn}\left(d^{2} \bar{t}_{q} / d \bar{\Omega}^{2}\right) \\
& \frac{d^{2} t_{a}}{d \bar{\Omega}^{2}}=\left[\frac{\bar{\Omega}}{\bar{t}_{a}^{2}} \frac{x}{\tau}\left(1-\frac{x}{\tau} \frac{t_{a}}{\bar{\Omega}}-\frac{\bar{k}_{q}^{2}}{\bar{\Omega}^{2}}\right)\right]+\left(\frac{2 \bar{\Omega} x / \tau}{\left[k_{q}^{2}\left(\bar{\Omega} / \bar{t}_{q}\right)^{2} / K^{4}\left(c / c_{s}\right)^{2}\left(\rho / \rho_{s}\right)(a / h)\right]+1-K_{0}^{2} / K_{1}^{2}}\right) \\
& \cdot\left\{\frac{\left(\bar{k}_{q}^{2} / \tilde{t}_{q}\right)^{2}}{K^{4}\left(c / c_{s}\right)^{2}\left(\rho / \rho_{s}\right)(a / h)}+\frac{2 x / \tau}{\bar{\Omega} \bar{t}_{q}}\left(1-\frac{\bar{t}_{a}}{\bar{\Omega}} \frac{x}{\tau}\right)\left(1-\frac{K_{0}^{2}}{K_{1}^{2}}\right)\right. \\
& \left.+\left(1-\frac{\bar{t}_{a}}{\bar{\Omega}} \frac{x}{\tau}\right)^{2}\left[\frac{K_{0}}{\bar{k}_{q} K_{1}}\left(1-\frac{K_{0}^{2}}{K_{1}^{2}}\right)-\frac{1}{\bar{k}_{\mathrm{e}}^{2}}\right]\right\}
\end{aligned}
$$

and

$$
K_{n}=K_{n}\left(\bar{k}_{a}\right) \quad(n=0,1)
$$




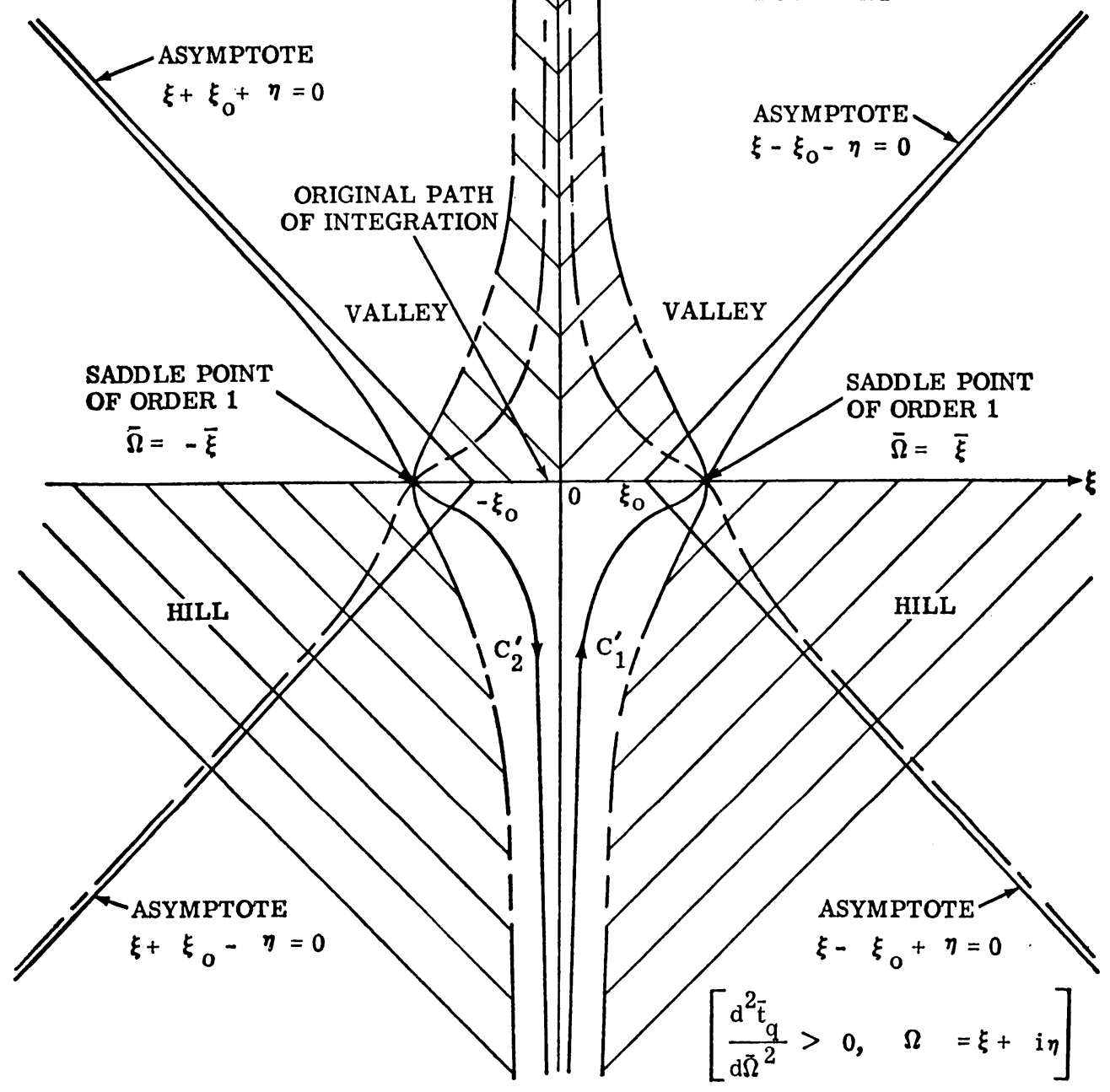

FIg. 3. Contour lines of $h(\Omega)$.

We have shown that the saddle points occur in pairs on the real axis. For positive $x / \tau$, Eq. (46) requires that the saddle point on the positive real axis, $\bar{\Omega}$, be associated with $\bar{t}_{a}$ while that on the negative real axis, $-\bar{Q}$, be associated with $-\bar{t}_{q}$. Also it has been pointed out that the deformed path $C^{\prime}$ passes through both saddle points. With the aid of Eqs. (16), (18), (46) and (50), it is easily shown that $\bar{t}_{q}, d^{2} \bar{t}_{q} / d \bar{\Omega}^{2}$ and $F^{(2 i)}(\bar{\Omega})$ are odd functions of $\bar{\Omega}$. Thus, by virtue of Eqs. (47) and (4S), we may write the expression for the contribution from the path $C^{\prime}=C_{1}^{\prime}+C_{2}^{\prime \prime}$ as follows: 


$$
\begin{aligned}
\frac{\sum_{a} I_{a}^{\prime}}{2 \pi i}=\left(\frac{2}{\pi \tau\left|d^{2} \bar{t}_{a} / d \bar{\Omega}^{2}\right|}\right)^{1 / 2} & \\
& \cdot\left\{F(\bar{\Omega}) \sin \psi+\sum_{1,3,5}^{\infty}(-1)^{(i-1) / 2}\left(\frac{1.3 \cdots(2 j-1) F^{(2 j)}(\bar{\Omega}) \cos \psi}{(2 j) !\left(\tau d^{2} \bar{t}_{a} / d \bar{\Omega}^{2}\right)^{i}}\right)\right. \\
& \left.+\sum_{2,4,6 \cdots}^{\infty}(-1)^{i / 2}\left(\frac{1.3 \cdots(2 j-1) F^{(2 j)}(\bar{\Omega}) \sin \psi}{(2 j) !\left(\tau d^{2} \bar{t}_{a} / d \bar{\Omega}^{2}\right)^{j}}\right)\right\}
\end{aligned}
$$

It should be pointed out that the above includes the contribution from both poles $\left(+\bar{t}_{a}\right)$ and $\left(-\bar{t}_{a}\right)$. If $\left|\tau d^{2} \bar{t}_{a} / d \bar{\Omega}^{2}\right|$ is sufficiently large, the above expression can be approximated by

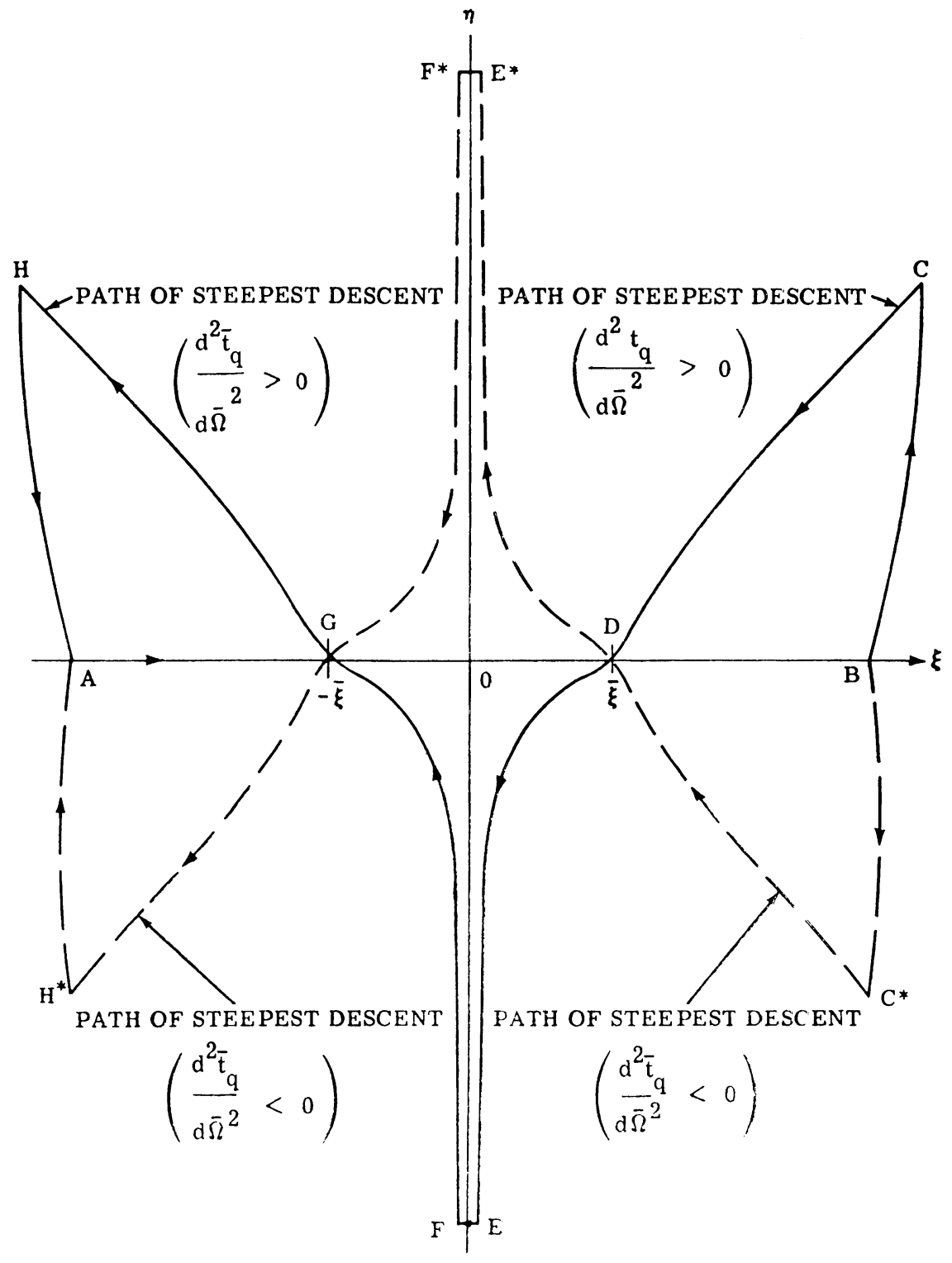

FIti. 4. Ieformed path of integration. 


$$
\frac{\sum_{a} I_{a}^{\prime}}{2 \pi i} \sim\left(\frac{2}{\pi \tau\left|d^{2} t_{a} / d \bar{\Omega}^{2}\right|}\right)^{1 / 2} F(\bar{\Omega}) \sin \psi .
$$

Contour integration of $w(x, \tau)$. Recall from Eq. (39) that the original path of integration has end points at $\pm \infty+i \alpha$. Thus from Fig. 3 we observe that the deformed steepest descent path $C^{\prime}$ has end points lying in the same valley as the original contour. Consequently, the integral of Eq. (39) can be evaluated by a contour integration along the path shown in Fig. 4. Cauchy's residue theorem is applied to the solid curve BCDEFGHA when $\left(d^{2} \bar{t}_{a} / d \bar{\Omega}^{2}\right)>0$, while the dotted curve $B C^{*} D E^{*} F^{*} G H^{*} A$ is to be used when $\left(d^{2} \bar{t}_{a} /\right.$ $\left.d \bar{\Omega}^{2}\right)<0$. Thus for $\left(d^{2} \bar{t}_{a} / d \bar{\Omega}^{2}\right)>0$, since the integral along $B C$ and $H A$ vanishes, we have

$$
\begin{aligned}
\frac{1}{2 \pi i} \int_{-\infty+i \alpha}^{\infty+i \alpha} \sum_{a} F(\Omega) \exp & {\left[i \tau\left(t_{a}(\Omega)-\frac{x}{\tau} \Omega\right)\right] d \Omega } \\
& =\frac{\sum_{a} I_{a}^{\prime}}{2 \pi i}+\sum\left[\operatorname{Res}_{(B C D B)}-\operatorname{Res}_{(D E F G D)}+\operatorname{Res}_{(G H A G)}\right]
\end{aligned}
$$

where, for example, $\operatorname{Res}_{(B C D B)}$ is the sum of the residues of the poles lying within the closed curve $B C D B$. A similar equation is obtained for $\left(d^{2} \bar{t}_{a} / d \bar{\Omega}^{2}\right)<0$.

The poles lying in the enclosed region are now obtained by letting the denominator of $F(\Omega)$ vanish (Eq. 18). Here both $\Omega$ and $t_{a}$ can be complex. But from Eq. (14) it can be shown that if $\operatorname{Im} \Omega=\eta \neq 0$ then $\operatorname{Im} t_{q}$ cannot vanish, and thus the residues will have an exponential term with negative nonvanishing real part which will decay rapidly for large $\tau$, and thus can be neglected. Note that poles having $\operatorname{Im} t_{a}<0$ have already been excluded (see Eq. (15) and the following statement). For $\eta=0, \operatorname{Im} t_{a}=0$, and if $\xi \neq 0$, then from Eq. (14) we observe that $\operatorname{Re} t_{a} \neq 0$, and that $k_{a}$ is real. But then the denominator of $F(\Omega)$ only vanishes when $\Omega=t_{a}=0$. However, recall that the function $F(\Omega)$ is associated with the poles obtained from Eq. (14) for which $\Omega=s=0$ is not a solution. Thus no singular points exist in the enclosed region. Thus Eq. (53) and the corresponding equation for $\left(d^{2} \bar{t}_{\mathrm{a}} / d \bar{\Omega}^{2}\right)$ $<0$ will, for $\left(d^{2} \bar{t}_{a} / d \bar{\Omega}^{2}\right) \neq 0$, reduce to

$$
\frac{1}{2 \pi i} \int_{-\infty+i \alpha}^{\infty+i \alpha} \sum_{a} F(\Omega) \exp \left[i \tau\left(t_{q}(\Omega)-\frac{x}{\tau} \Omega\right)\right]=\frac{\sum_{a} I_{a}^{\prime}}{2 \pi i} .
$$

If $\left|\tau d^{2} \bar{t}_{a} / d \bar{\Omega}^{2}\right|$ is "sufficiently large", then by inserting Eq. (52) into Eq. (54) and substituting the resulting expression into Eq. (38), we obtain

$$
\begin{aligned}
& \frac{w(x, \tau)}{\frac{a}{h} \frac{p_{0}}{E}} \sim\left(\frac{2}{\pi \tau\left|d^{2} \bar{t}_{a} / d \bar{\Omega}^{2}\right|}\right)^{1 / 2} F(\bar{\Omega}) \sin \psi \\
& \quad-\frac{1}{4 \pi^{2} i} \int_{-\infty+1 \alpha}^{\infty+\imath \alpha} \frac{e^{i \Omega(r-x)}}{\Omega} d \Omega \int_{\pi}^{-\pi} \frac{d \theta}{\frac{\Omega^{4}}{4 K^{4}}+1-\Omega^{2}\left(\frac{c}{c_{s}}\right)^{2}\left[1+\left(\frac{\rho}{\rho_{s}}\right)\left(\frac{a}{h}\right) \frac{K_{0}\left(k_{+}\right)}{k_{+} K_{1}\left(k_{+}\right)}\right]} \\
& +\frac{1}{4 \pi^{2} i} \int_{-\infty+2 \alpha}^{\infty+\imath \alpha} \frac{e^{-i \Omega(\tau+x)}}{\Omega} d \Omega \int_{\pi}^{-\pi} \frac{\frac{\Omega^{4}}{4 K^{4}}+1-\Omega^{2}\left(\frac{c}{c_{s}}\right)^{2}\left[1+\left(\frac{\rho}{\rho_{s}}\right)\left(\frac{a}{h}\right) \frac{K_{0}\left(k_{-}\right)}{k_{-} K_{1}\left(k_{-}\right)}\right]}{}
\end{aligned}
$$


Note that as $|k| \rightarrow 0,\left[K_{0}(k) / k K_{1}(k)\right] \approx \ln \rightarrow-\infty$, and that thus the two integrands of Eq. (55) are finite (indeed, as $\epsilon \rightarrow 0$, they vanish) for all $\Omega \neq 0$. A simple pole exists, however, at $\Omega=0$. By using the residue theorem, it is easily shown that for $x>0$ and $\tau-x>0$, the first integral expression vanishes, while the second integral is equal to +1 . For $\tau-$ $x<0$ the first integral expression is equal to -1 , while the second is +1 . Similar results are obtained for $x<0$. We thus may rewrite Eq. (55) in its final form

$$
\frac{w(x, \tau)}{a p_{0} /(h E)} \sim H(\tau-|x|)+\left(2 / \pi \tau\left|\frac{d^{2} \bar{t}_{q}}{d \bar{\Omega}^{2}}\right|\right)^{1 / 2} F(\bar{\Omega}) \sin \psi .
$$

It should be pointed out that if $d^{2} \bar{t}_{a} / d \bar{\Omega}^{2}$ is small, then $\left|\tau d^{2} \bar{t}_{a} / d \bar{\Omega}^{2}\right|$ may, for some given $\tau$, not be "sufficiently large", and the third order solution must be used.

Third order solution. If, in evaluating $j=\int_{\gamma} e^{\tau h(\Omega)} d \Omega$ by the method of steepest descent, the magnitude of $\left|\tau h^{\prime \prime}(\bar{\Omega})\right|$ is not sufficiently large, it is necessary to use a third order solution [13], i.e., one in which the third derivative of $h(\Omega)$ is included in the Taylor series expansion of $h(\Omega)$ about the saddle point. Thus noting that $h^{\prime \prime}(\bar{\Omega})=i\left(d^{2} \bar{t}_{q} / d \bar{\Omega}^{2}\right)$ and $h^{\prime \prime \prime}(\bar{\Omega})=i\left(d^{3} \bar{t}_{a} / d \bar{\Omega}^{3}\right)$, and approximating $F(\Omega)$ by $F(\bar{\Omega})$, we obtain, after some algebraic manipulation [12], the following:

$$
\left.I_{a}^{\prime}\right|_{\bar{\Omega}}=F(\bar{\Omega})\left[6 / \tau\left(d^{3} \bar{t}_{a} / d \bar{\Omega}^{3}\right)\right]^{1 / 3} e^{i \varphi} \int_{-\infty}^{\infty} e^{i\left(\omega^{0}-\sigma \omega\right)} d \omega
$$

where

$$
g=\frac{\tau}{2} \frac{\left(d^{2} t_{a} / d \bar{\Omega}^{2}\right)^{2}}{\left(d^{3} t_{a} / d \bar{\Omega}^{3}\right)}\left(\frac{6}{\tau\left(d^{3} \bar{t}_{a} / d \bar{\Omega}^{3}\right)}\right)^{1 / 3} ; \quad \varphi=\tau \bar{t}_{a}-x \bar{\Omega}+\frac{\tau}{3} \frac{\left(d^{2} \bar{t}_{a} / d \bar{\Omega}^{2}\right)^{3}}{\left(d^{3} \bar{t}_{a} / d \bar{\Omega}^{3}\right)^{2}} .
$$

As the saddle point is on the real axis, $g$ is real, and

$$
\int_{-\infty}^{\infty} e^{i\left(\omega^{3}-\vartheta \omega\right)} d \omega=2 \int_{0}^{\infty} \cos \left(\omega^{3}-\zeta \omega\right) d \omega
$$

Then Eq. (57) can be rewritten as [14]

$$
\left.I_{a}^{\prime}\right|_{\bar{\Omega}}=2 \pi F(\bar{\Omega})\left(\frac{2}{\tau\left(d^{3} \bar{t}_{a} / d \bar{\Omega}^{3}\right)}\right)^{1 / 3} e^{i \varphi} \mathrm{Ai}\left[\frac{-\frac{\tau}{2}\left(d^{2} \bar{t}_{q} / d \bar{\Omega}^{2}\right)^{2}}{\left(d^{3} \bar{t}_{q} / d \bar{\Omega}^{3}\right)}\left(\frac{2}{\tau\left(d^{3} \bar{t}_{q} / d \bar{\Omega}^{3}\right)}\right)^{1 / 3}\right]
$$

where $\mathrm{Ai}(z)=$ the Airy function of $z$. But the saddle point occurs in pairs and $F(\bar{\Omega}), \bar{t}_{a}$, $d^{2} \bar{t}_{a} / d \bar{\Omega}^{2}$ and $\varphi$ are odd functions of $\bar{\Omega}$ and $d^{3} \bar{t}_{\alpha} / d \bar{\Omega}^{3}$ is an even function of $\bar{\Omega}$; thus we finally arrive at

$$
\frac{\sum_{a} I_{a}^{\prime}}{2 \pi i}=2 F(\bar{\Omega})\left(\frac{2}{\tau\left(d^{3} \bar{t}_{a} / d \bar{\Omega}^{3}\right)}\right)^{1 / 3} \sin \varphi \mathrm{Ai}\left[-\frac{-\frac{\tau}{2}\left(d^{2} \bar{t}_{a} / d \bar{\Omega}^{2}\right)^{2}}{\left(d^{3} \bar{t}_{a} / d \bar{\Omega}^{3}\right)}\left(\frac{2}{\tau\left(d^{3} \bar{t}_{a} / d \bar{\Omega}^{3}\right)}\right)^{1 / 3}\right] .
$$

Substituting Eq. (60) into Eq. (54), we have for the equivalent third order solution of Eq. (56)

$$
\frac{w(x, \tau)}{\left(\alpha^{\prime} / h\right)\left(p_{0} / E\right)} \sim H(\tau-|x|)+2 F(\bar{\Omega})\left(\frac{2}{\tau\left(d^{3} \bar{t}_{a} / d \bar{\Omega}^{j}\right)^{-5}}\right)^{1 / 3} \sin \varphi \mathrm{Ai}(-z)
$$

where

$$
z=\frac{\tau}{2}\left(\frac{d^{2} t_{q}}{d \bar{\Omega}^{2}}\right)^{2}\left(\frac{2}{\tau\left(d^{3} \bar{t}_{q} / d \bar{\Omega}^{3}\right)}\right)^{1 / 3}\left(\frac{d^{3} \bar{t}_{q}}{d \bar{\Omega}}\right)^{-1}
$$


VI. Numerical calculations. It has been shown that for given $x / \tau$ the three simultaneous equations (42)-(44) lead to a solution for the saddle point, $\bar{\Omega}$, and for $\bar{t}_{a}$ and $\bar{k}_{q}$. The solution of these equations can be attained by first assuming a value of $\bar{k}_{a}$. By utilizing Eq. (44), $\bar{t}_{4}$ can then be determined, and then from Eq. (43) $\bar{\Omega}$ obtained. Finally, $x / \tau$ is determined from Eq. (42). It should be noted that the $\bar{k}_{q}$ 's are chosen so as to make $\bar{t}_{a}$ real. The radial deflection $w$ is then determined from either Eq. (56) or Eq. (61), the second and third order solutions, respectively. The numerical investigation was carried out for a steel circular cylindrical shell submerged in water, having a radius-thickness ratio of $87 \frac{1}{3}$. With these physical properties, we have: $a_{/} / h=87 \frac{1}{3}, \nu=0.3,\left(c / c_{\mathrm{s}}\right)^{2}=.08815, \rho / \rho_{\mathrm{s}}=$ .13054 . It should be noted that for each value of $x / \tau, \bar{k}_{a}$ is double valued. Associated with the lower values are the low frequency modes, which are of large amplitude, while the higher values yield the low amplitude high frequency modes [15]. For the low frequency modes $\left(d^{2} \bar{t}_{a} / d \bar{\Omega}^{2}\right)$ is rather small and therefore a third order solution is required, while for the high frequency modes $\left(d^{2} \bar{t}_{a} / d \bar{\Omega}^{2}\right)$ is "sufficiently large", and the second order solutions suffice.

VII. Discussion of results. The reflection ratio, $w_{/}^{\prime}\left(a p_{0} / h E\right)$, obtained from the exact (asymptotic), plane wave (Appendix B), and static (Appendix C) solutions has been plotted as a function of the position parameter $\sigma$ in Figs. 5 to 11. In Figs. 5, 6, and 7 the large parameter $\tau$ of the asymptotic solution was taken to be equal to 1000 , while $\tau=500$ in Figs. 8, 9, and 10. Finally, all results for the deflection ratio are presented in Fig. 11 for $-.14 \leq \sigma \leq .10$.

It should be noted that in the region behind the wave front $(\sigma>.3)$, the plane wave and static solutions are almost identical and equal to unity. However, the asymptotic solution exhibits a large amplitude, low frequency, damped oscillation about the static equilibrium position. The analytical investigation of the asymptotic solution suggests

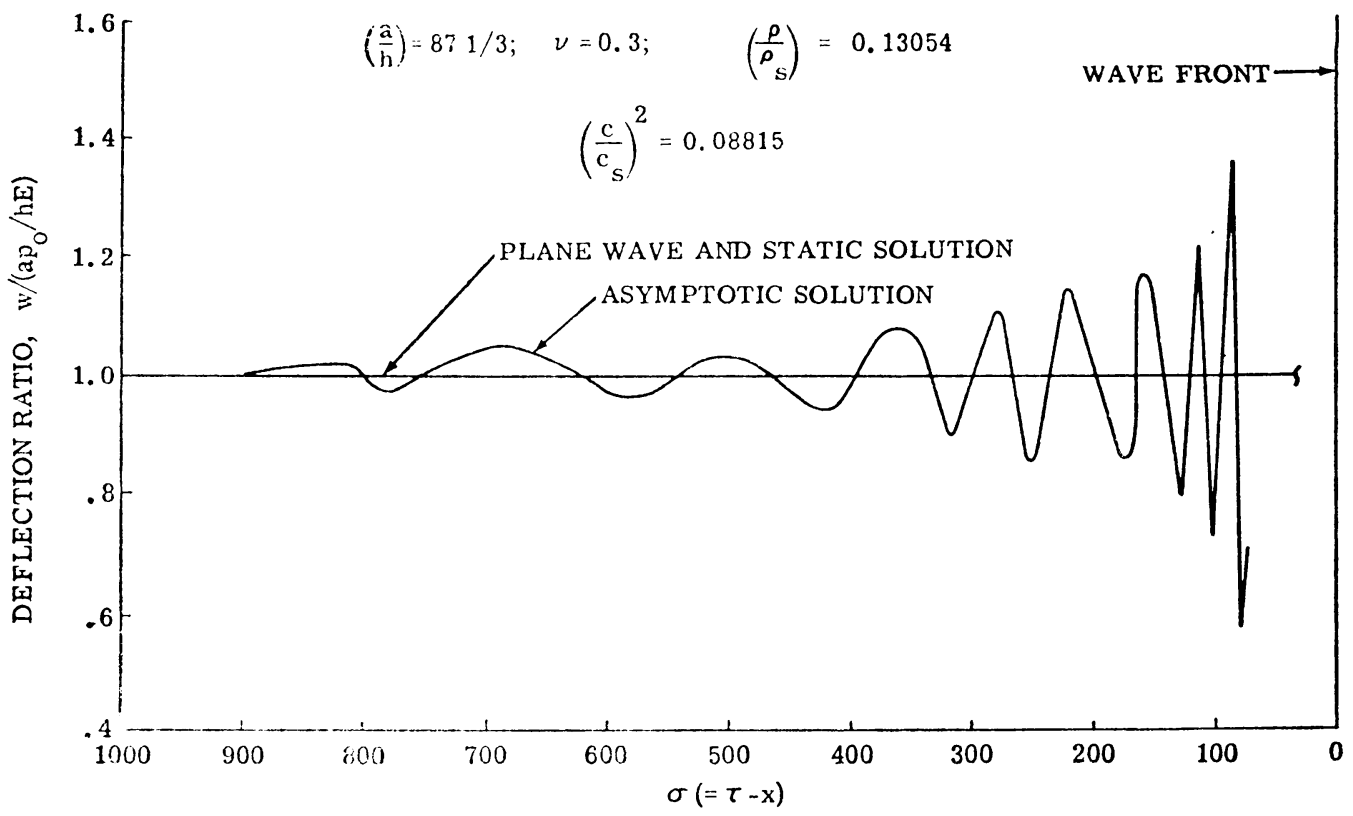

Fis. 5. Deflection ratio $(\tau==1000 ; \sigma \geq 76)$. 
that the solution can be decomposed into a high frequency, small amplitude mode and a low frequency, large amplitude mode [15]. It is the contribution from the latter component that yields the observed oscillations.

In front of the wave the asymptotic and static solutions are almost identical (Fig. 11) for $\sigma<-.14$, and it is seen that in this region the deflection vanishes.

Excellent agreement on the general forms and amplitudes of the oscillations has been obtained by using $\tau=500$ and 1000. Furthermore, results obtained by choosing $\tau=2000$, although not plotted, agreed quite well with those obtained by using $\tau=1000$, thereby lending credence to the assumption that the asymptotic solution is valid for $\tau>1000$.

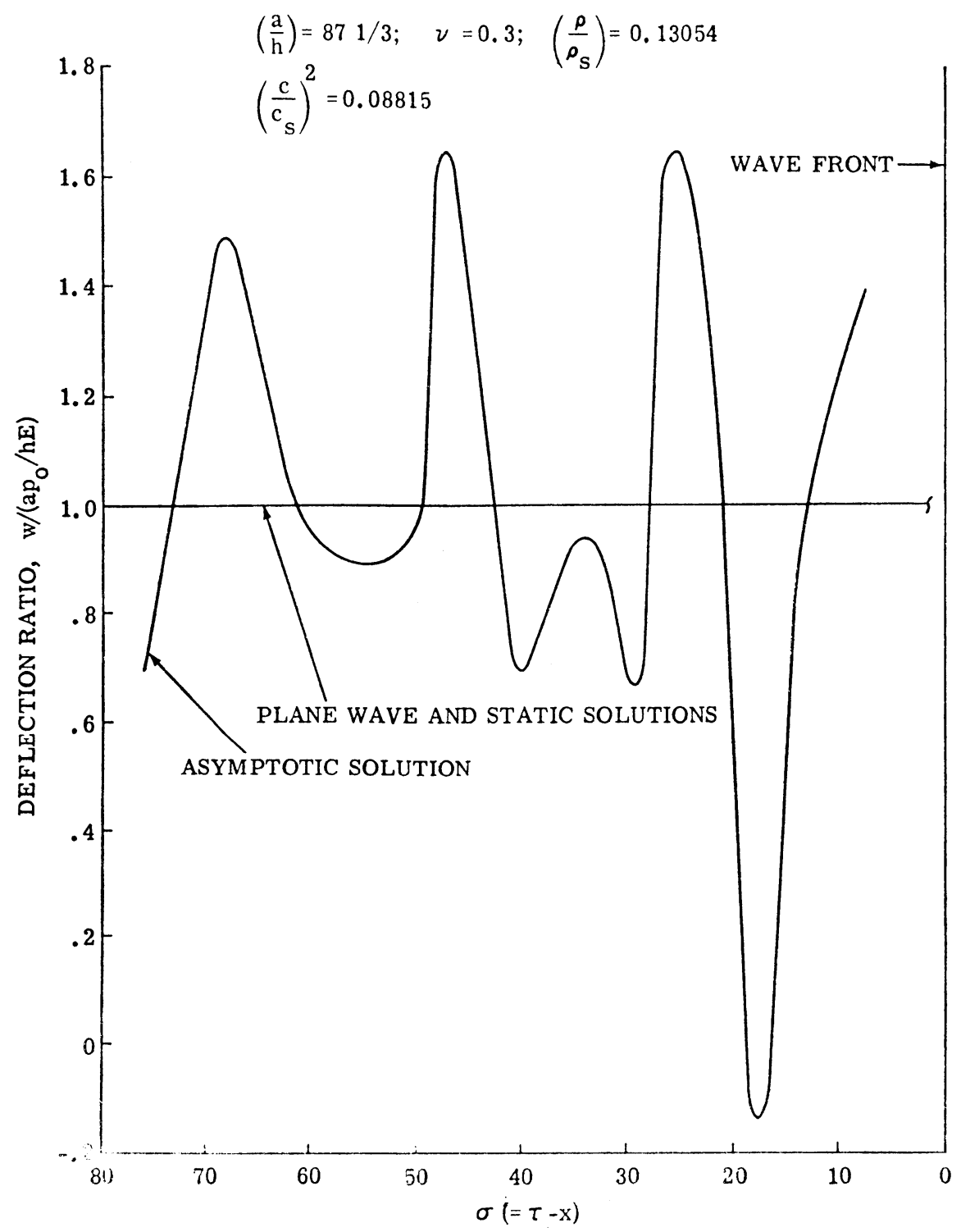

Fig. 6. Deflection ratio $(r=1000 ; 8.1 \leq \sigma \leq 76)$. 


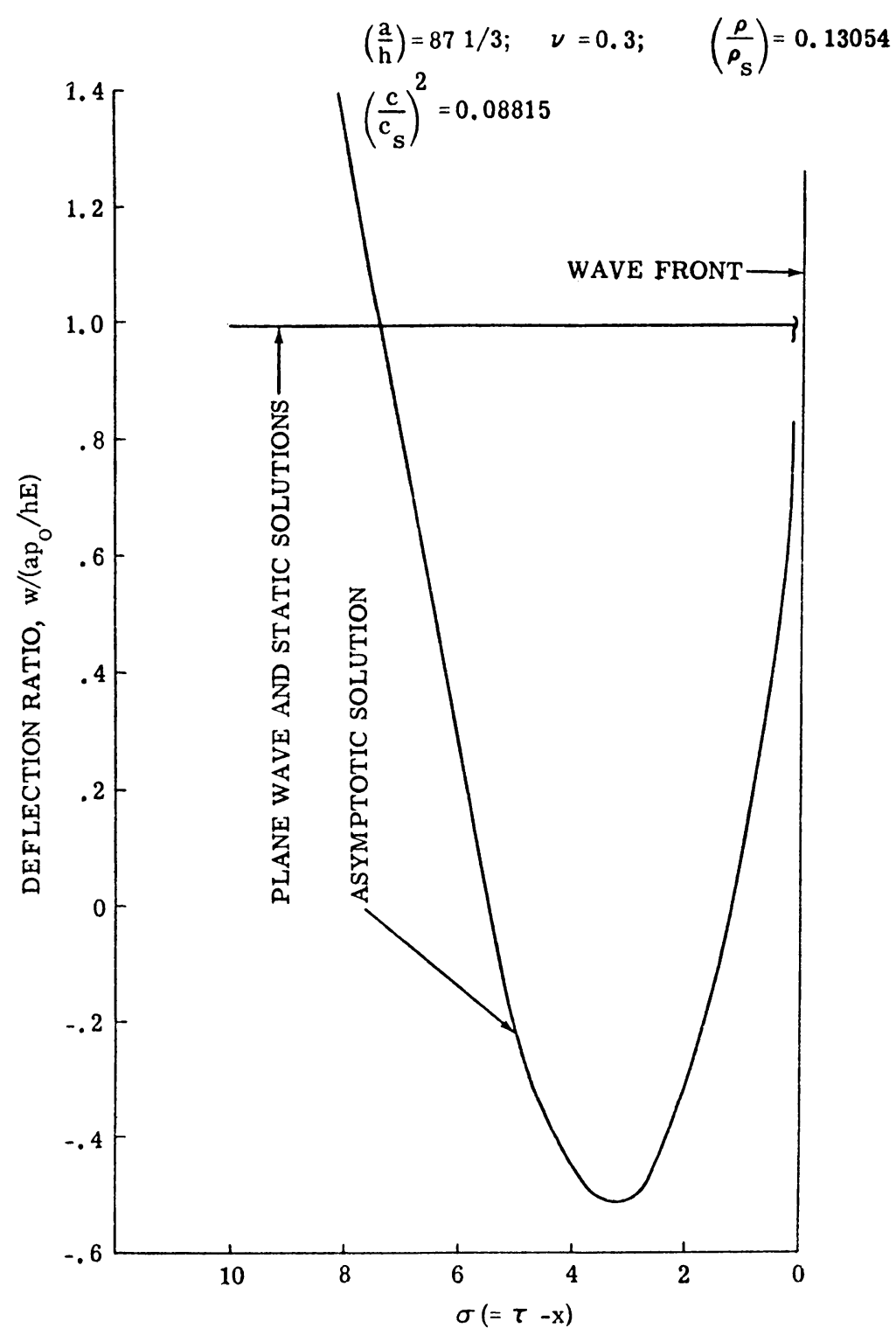

Fig. 7. Deflection ratio $(\tau=1000 ; 0.1 \leq \sigma \leq 8.1)$.

Appendix A

Investigation of contour of $h(\Omega)$. Consider the behavior of $h(\Omega)$ in the immediate vicinity of the saddle point, where

$$
i(\Omega)-h(\bar{\Omega})=\frac{(\Omega-\bar{\Omega})^{n}}{n !} h^{(n)}(\bar{\Omega})=P+i Q
$$

By setting

$$
\Omega-\bar{\Omega}=|\Omega-\bar{\Omega}| e^{i \theta} \quad \text { and } \quad h^{(n)}(\bar{\Omega}) / n !=\left|h^{(n)}(\bar{\Omega}) / n !\right| e^{i \alpha}
$$




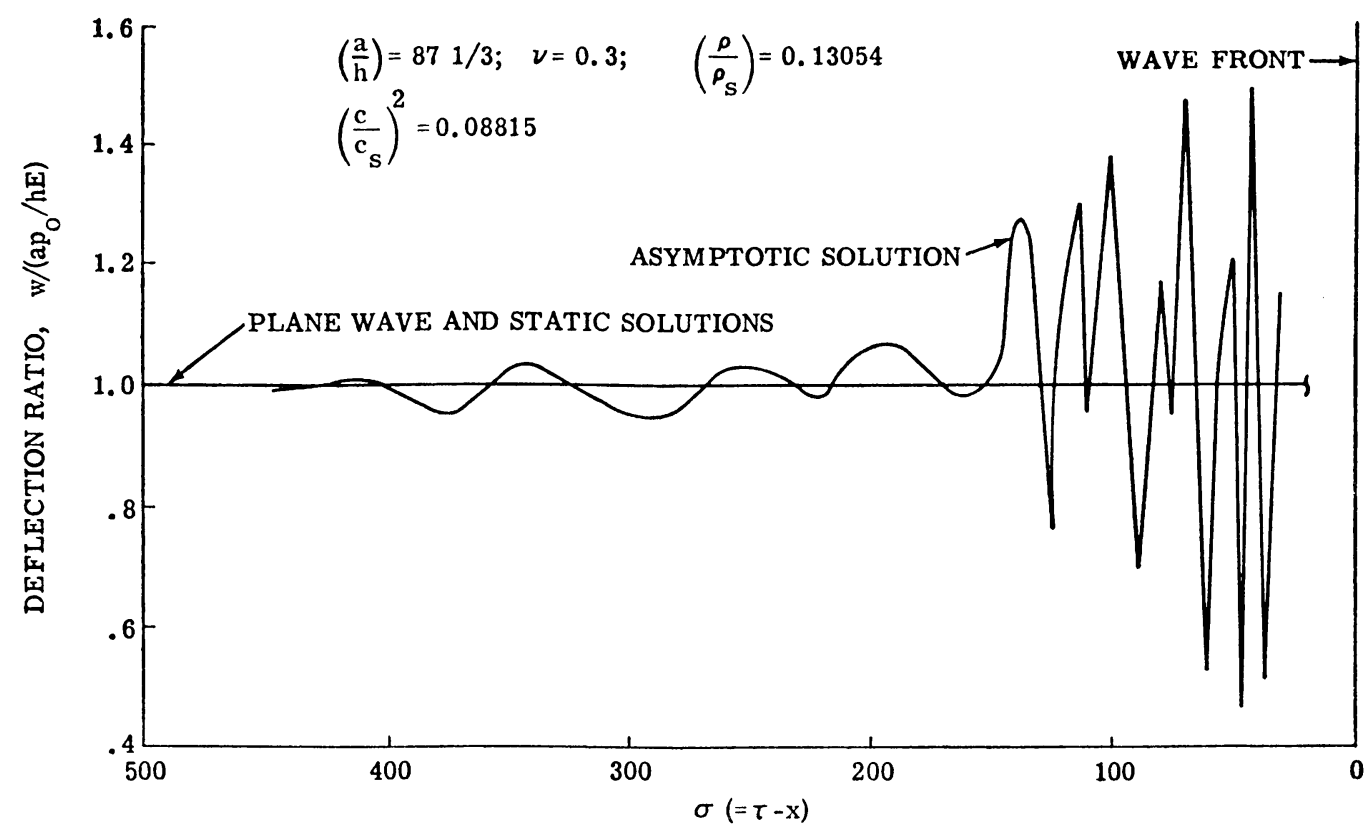

FIG. 8. Deflection ratio $(\tau=500 ; \sigma \geq 34)$.

$$
P=\left|h^{(n)}(\bar{\Omega}) / n !\right||\Omega-\bar{\Omega}|^{n} \cos (n \theta+\alpha) ; \quad Q=\left|h^{(n)}(\bar{\Omega}) / n !\right||\Omega-\bar{\Omega}|^{n} \sin (n \theta+\alpha) .
$$

For boundary, steepest descent and steepest ascent curves, $P$ is respectively zero, the largest possible negative value and the largest possible positive value. The corresponding directions in the vicinity of the saddle point $\bar{\Omega}$ are given in Table I.

We have shown that the saddle points associated with the integral of Eq. (39) occur in pairs and are located on the real axis of the complex $\Omega(\xi, \eta)$-plane. Let them be denoted by $\bar{\Omega}= \pm \bar{\xi}$ where $\bar{\xi}$ is a nonnegative real quantity. When $\bar{\Omega}=\bar{\xi},\left(d^{2} \bar{t}_{a} / d \bar{\Omega}^{2}\right)>0$, but since $h^{\prime \prime}(\bar{\Omega})=i\left(d^{2} \bar{t}_{a} / d \bar{\Omega}^{2}\right), n=2, \alpha=\pi / 2$. Therefore in the vicinity of $\bar{\Omega}=\bar{\xi}$ there are two distinct directions of descent given by $\theta_{D}=\pi / 4,5 \pi / 4$, and two distinct directions of ascent, $\theta_{A}=-\pi / 4,3 \pi / 4$, and the following directions for the boundary curves $\theta_{B}=0, \pi / 2, \pi, 3 \pi / 2$ in the vicinity of $\bar{\Omega}=-\bar{\xi}, d^{2} \bar{t}_{a} / d \bar{\Omega}^{2}<0$, and the directions of descent are $\theta_{D}=3 \pi / 4,7 \pi / 4$, those of ascent are $\theta_{A}=\pi / 4,5 \pi / 4$ and the directions for the boundary curves are $\theta_{B}=$ $\pi / 2, \pi, 3 \pi / 2,2 \pi$.

TABLE I

Directions of Steepest Descent and Ascent and Boundary

Curves at a Saddle Point of Order $n-1$

curve

steepest descent steepest ascent boundary
Direction, $\theta$, in the vicinity of the saddle point

$-\alpha / n+(2 q+1) \pi / n$

$-\alpha / n+2 q \pi / n$

$-\alpha / n+\left(q+\frac{1}{2}\right) \pi / n$ 


$$
\begin{aligned}
& \left(\frac{a}{h}\right)=871 / 3 ; \quad \nu=0.3 ; \quad\left(\frac{\rho}{\rho_{S}}\right)=0.13054 \\
& \left(\frac{c}{c_{S}}\right)^{2}=0.08815
\end{aligned}
$$

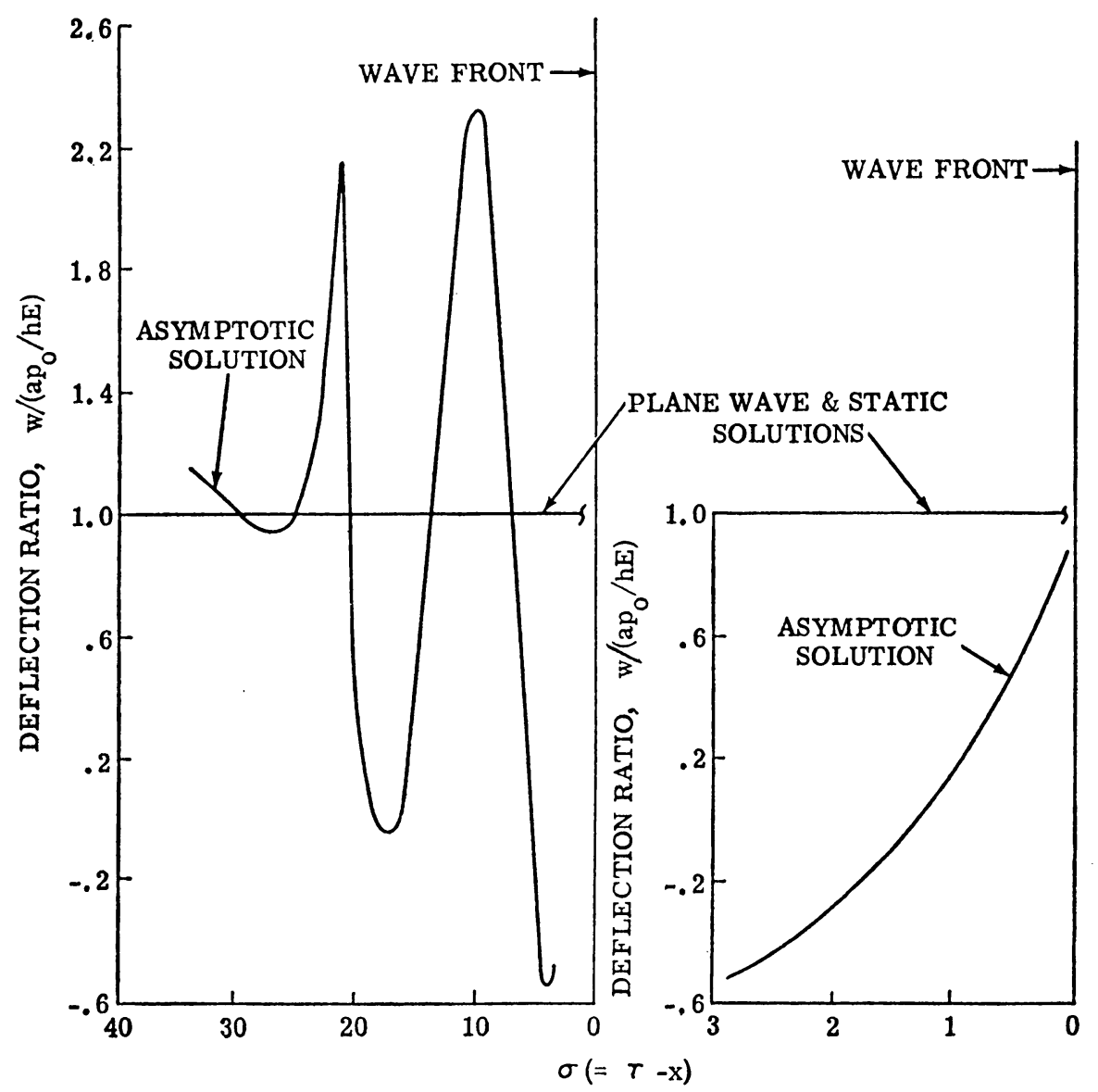

FIG. 9. Deflection ratio

FIG. 10. Deflection ratio

$(\tau=500 ; 2.9 \leq \sigma \leq 34)$.

$(\tau=500 ; 0.1 \leq \sigma \leq 2.9)$.

Steepest paths do not intersect.

Let

$$
\Omega=\xi+i \eta, t_{a}(\Omega)=\operatorname{Re}\left[t_{\alpha}\right]+i \operatorname{Im}\left[t_{a}\right], \text { and } h(\Omega)=U(\xi, \eta)+i V(\xi, \eta) .
$$

On steepest paths

$$
U(\xi, \eta) \lessgtr \bar{U}(\bar{\xi}, \bar{\eta})=(x / \tau) \bar{\eta}-\operatorname{Im}\left[\bar{t}_{a}\right]=0
$$

where $<$ and $>$ are used for descent and ascent paths respectively, and the bar represents the values at the saddle point. Furthermore, on the steepest paths

$$
V(\xi, \eta)=\operatorname{Re} t_{a}-(x / \tau) \xi=\operatorname{Re} \bar{t}_{a}-(x / \tau) \bar{\xi} .
$$

But the saddle points occur in pairs, i.e., $\bar{\Omega}= \pm \bar{\xi}$, and thus Eq. (A-6) can be rewritten as

$$
\left(\operatorname{Re} t_{a}-(x / \tau) \xi\right)_{+}=\bar{t}_{a}(\bar{\xi})-(x / \tau) \bar{\xi}
$$




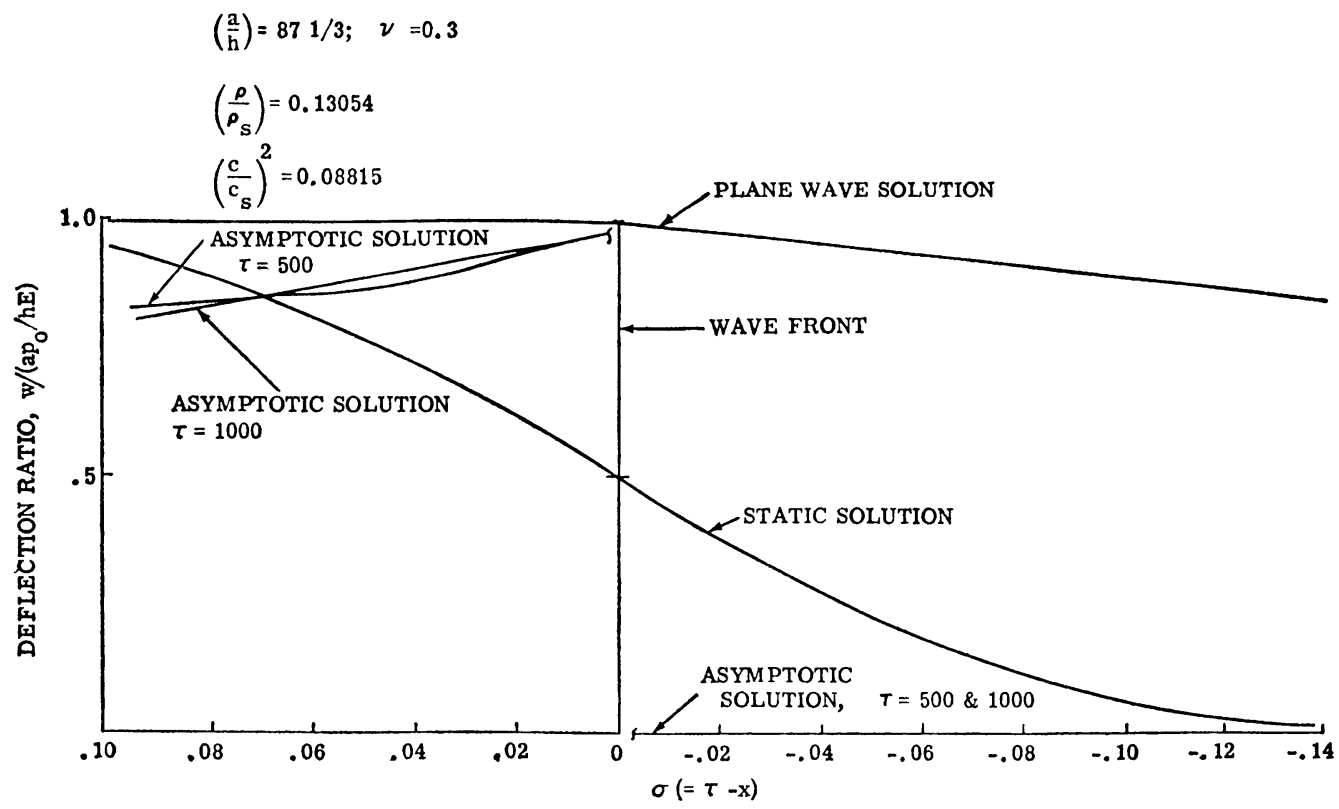

FIG. 11. Deflection ratio (vicinity of wave front) (asymptotic solution invalid for $|\sigma| \leq .00003$ ).

and

$$
\left(\operatorname{Re} t_{a}-(x / \tau) \xi\right)_{-}=\bar{t}_{a}(-\bar{\xi})-(x / \tau)(-\bar{\xi})
$$

where the subscripts + and - correspond to the paths passing through the saddle points at $\bar{\xi}$ and $-\bar{\xi}$, respectively. Recalling that $\bar{t}_{a}(\bar{\xi})=-\bar{t}_{a}(-\bar{\xi})$, we observe that the above two equations are equal in magnitude but of opposite sign, and thus the paths through $\pm \xi$ do not intersect in the finite region.

Asymptotes for steepest paths. On the steepest paths the following equation must be satisfied:

$$
\Omega^{4}+4 K^{4}-4 K^{4} t_{a}^{2}\left(\frac{c}{c_{s}}\right)^{2}\left[1+\left(\frac{\rho}{\rho_{s}}\right)\left(\frac{a}{h}\right) \frac{K_{0}\left(k_{q}\right)}{k_{q} K_{1}\left(k_{q}\right)}\right]=0
$$

where

$$
k_{a}=\left[\Omega^{2}-t_{a}^{2}\right]^{1 / 2}
$$

$\Omega$ and $t_{a}$ are, in general, complex.

To determine the asymptotes of the path, let $|\Omega| \rightarrow \infty$; and first let us assume that $t_{q}=\Omega$ as $|\Omega| \rightarrow \infty$. Then $k_{q} \rightarrow 0$ and Eq. (A-9) reduces to

$$
t_{a}^{4}\left[1+4 K^{4}\left(c / c_{s}\right)^{2}\left(\rho / \rho_{s}\right)(a / h) \ln k_{a} / t_{a}^{2}\right]=0,
$$

but

$$
\lim _{t_{a} \rightarrow \Omega \rightarrow \infty}\left(\ln k_{d} / t_{c}^{2}\right)=\lim _{k_{Q} \rightarrow 0}\left(-1 / 2 k_{0}^{2}\right) \rightarrow-\infty .
$$

Thus Eq. (A-11) is not satisfied nnd we can rule out our first assumption. Consider instead that $t_{a} \neq \Omega$ as $|\Omega| \rightarrow \infty$. For this case, $\left|k_{a}\right| \rightarrow \infty$, and Eq. (A-9) reduces to $\Omega^{4}-$ 
$4 K^{4} t_{a}^{2}\left(c / c_{s}\right)^{2}=0$ and thus

$$
t_{\mathrm{q}}= \pm \frac{\Omega^{2}}{2 K^{2}\left(c / c_{s}\right)}= \pm \frac{\xi^{2}-\eta^{2}+2 i \xi \eta}{2 K^{2}\left(c / c_{s}\right)} .
$$

Finally then

$$
\operatorname{Re} t_{a}= \pm \frac{\xi^{2}-\eta^{2}}{2 K^{2}\left(c / c_{s}\right)} ; \quad \operatorname{Im} t_{a}= \pm \frac{\xi \eta}{K^{2}\left(c / c_{s}\right)} .
$$

Substituting Eq. (A-13) into Eq. (A-6) yields

$$
\pm \frac{\xi^{2}-\eta^{2}}{2 K^{2}\left(c / c_{s}\right)}-\frac{x}{\tau} \xi=\operatorname{Re} t_{a}-\frac{x}{\tau} \bar{\xi}
$$

or

$$
\left(\xi \pm \xi_{0}\right)^{2}-\eta^{2}= \pm C_{1}
$$

where $\xi_{0}$ and $C_{1}$ are functions of $x / \tau$ since $\bar{t}_{a}$ and $\bar{\xi}$ are functions of $x / \tau$. For given $x / \tau, C_{1}$ and $\xi_{0}$ are constants, and the asymptotes are

$$
\xi \pm \xi_{0} \pm \eta=0
$$

which are, of course, the asymptotes of the steepest paths. By observing Eqs. (44) and (45), which also hold for the equivalent unbarred quantities, it can be seen that if $\operatorname{Im} \Omega=$ $\eta \neq 0$, then $\operatorname{Im} t_{a} \neq 0$. Then, by virtue of Eq. (A-13), $\xi$ never vanishes when $\eta \neq 0$. Thus the steepest path never crosses the imaginary $\eta$ axis. Accordingly, with the aid of Eq. (A-13), it is seen that the imaginary axis, $\xi=0$, also serves as an asymptote for the steepest path.

Boundary curves. On a boundary curve $U(=\operatorname{Re} h)$ is constant. Thus on the boundary curve passing through the saddle points $\bar{\Omega}= \pm \bar{\xi}$ we have (see Eq. (A-5))

$$
U(\xi, \eta)=(x / \tau) \eta-\operatorname{Im} t_{a}=0 .
$$

Upon substituting Eq. (A-13) into Eq. (A-17), we obtain the asymptotes of the boundary curves, i.e.,

$$
\eta\left(\frac{x}{\tau} \mp \frac{\xi}{K^{2}\left(c / c_{*}\right)}\right)=0 .
$$

Thus $\eta=0$ and $\xi= \pm\left(x_{/}^{\prime} \tau\right) K^{2}\left(c / c_{s}\right)$ are the asymptotes of our boundary curves.

Contour lines of $h(\Omega)$. After determining the locations of the saddle points, the directions of the steepest paths, the boundary curves in the vicinity of the saddle points and the asymptotes of the steepest paths and boundary curves, we may sketch the contours of the surface of $h(\Omega)$. Fig. 3. shows this surface for $\left(d^{2} \bar{t}_{a} / d \bar{\Omega}^{2}\right)>0$. However Fig. 3 is also valid for $\left(d^{2} \bar{t}_{a} / d \bar{\Omega}^{2}\right)<0$, provided that descent and ascent curves as well as hill and valley regions are interchanged.

\section{Appendix B}

Steady-state response-plane wave approximation. Carrier [6] investigated the steady-state response of a submerged, infinitely long, circular cylindrical shell subjected to an axially progressing acoustic wave. He used a plane wave approximation for the acoustic reflected wave and included the rotatory inertia terms in the shell theory. How- 
ever, it was pointed out that the rotatory inertia terms had no appreciable effect on the results even in high-speed impact problems. Thus, in order to compare the steady-state results to the asymptotic solution of the problem considered here, the Carrier solution has been repeated by using the Donnell shell theory. In so doing the final uncoupled shell equation in which the acoustic field has been accounted for by the plane wave approximation is

$$
w^{\mathrm{IV}}+4 K^{4} w+4 K^{4}\left(\frac{c}{c_{s}}\right)^{2} w^{\prime \prime}-4 K^{4}\left(\frac{\rho}{\rho_{s}}\right)\left(\frac{a}{h}\right)\left(\frac{c}{c_{s}}\right)^{2} w^{\prime}=4 K^{4} \frac{a p_{0}}{h E} H(\sigma)
$$

where $w^{(n)}=d^{n} w / d \sigma^{n}$. The desired solution for $w$ is

$$
\begin{aligned}
\frac{w}{(a / h)\left(p_{0} / E\right)} & =C_{1} e^{\lambda_{1} \sigma}+C_{2} e^{\lambda_{2} \sigma} & \text { for } \quad \sigma \leq 0 \\
& =1-C_{3} e^{\lambda_{3} \sigma}-C_{4} e^{\lambda_{4} \sigma} & \text { for } \quad \sigma \geq 0
\end{aligned}
$$

where $\lambda_{1}, \lambda_{2}$ have positive real parts and $\lambda_{3}, \lambda_{4}$ have negative real parts. The constants $C_{i}(j=1,2,3,4)$ of integration are to be determined by the continuity condition, $w, w^{\prime}, w^{\prime \prime}$ and $w^{\prime \prime \prime}$ at $\sigma=0$. By doing so, we have

$$
C_{i}=\prod_{k \neq j} \frac{\lambda_{k}}{\lambda_{k}-\lambda_{j}}
$$

and $\lambda_{i}, j=1,2,3,4$ are the four roots obtained from

$$
\lambda^{4}+4 K^{4}\left[\left(c / c_{s}\right)^{2} \lambda^{2}-\left(\rho / \rho_{s}\right)(a / h)\left(c / c_{s}\right)^{2} \lambda+1\right]=0 .
$$

Appendix C

Static solution. The static solution of the Carrier problem is determined from the following differential equation:

$$
d^{4} w /\left(d \sigma^{4}\right)+4 K^{4} w=4 K^{4} a p_{0} /(h E) H(\sigma) .
$$

For a cylindrical shell of infinite length, the solution is

$$
\begin{aligned}
\frac{w}{(a / h)\left(p_{0} / E\right)} & =1+e^{-K \sigma}\left(C_{1} \cos K \sigma+C_{2} \sin K \sigma\right) & & \text { for } \quad \sigma \geq 0 \\
& =e^{K \sigma}\left(C_{3} \cos K \sigma+C_{4} \sin K \sigma\right) & & \text { for } \quad \sigma \leq 0 .
\end{aligned}
$$

$C_{i}, j=1,2,3,4$, are constants of integration to be determined by the continuity conditions for $w, w^{\prime}, w^{\prime \prime}, w^{\prime \prime \prime}$ at $\sigma=0$. By doing so, we have

$$
\begin{array}{rlrl}
\frac{w}{(a / h)\left(p_{0} / E\right)} & =1-\frac{1}{2} e^{-K \sigma} \cos K \sigma & & \text { for } \quad \sigma \geq 0, \\
& =\frac{1}{2} e^{K \sigma} \cos K \sigma & \text { for } \quad \sigma \leq 0 .
\end{array}
$$

\section{REFERENCES}

[1] R. D. Mindlin and H. II. Bieich, Response of an elastic cylindrical shell to a transverse, step shock wave, J. Appl. Mech. 20, 189-195 (1953)

[2] A.I. L. Baron, The rcsponse of a cylindrical shell to a transverse shock wave, Proc. Second U.S. National Congress of Applied Mechanics, 1954, pp. 201-212

[3] J. H. Haywood, Response of an elastic cylindrical shell to a pressure pulse, Quart. J. Mech. Appl. Math. 11, 129-141 (1958) 
[4] H. Herman and J. M. Klosner, Transient response of a periodically supported cylindrical shell immersed in a fluid medium, J. Appl. Mech. Ser. E 32, 562-568 (1965)

[5] J. W. Berglund and J. M. Klosner, Interaction of a ring-reinforced shell and a fluid medium, J. Appl. Mech. Ser. E 35, 139-147 (1968)

[6] G. F. Carrier, The response of a submerged cylindrical shell to an axially propagating acoustic wave, Contract N7 ONr-35810, no. B 11-19/7, Brown University, Providence, R.I., 1953

[7] F. Herrmann and J. E. Russell, Forced motions of shells and plates surrounded by an acoustic fluid, Proc. Sympos. Theory of Shells in Honor of Lloyd Hamilton Ionnell, University of Houston, Houston, Texas, 1967, pp. 311-339

[8] A. Erdélyi, Asymptotic expansions, Dover, New York, 1956

[9] G. F. Carrier, M. Krook and C. E. Pearson, Functions of a complex variable, McGraw-Hill, New York, 1966

[10] H. Jeffreys and B. S. Jeffreys, Methods of mathematical physics, 2nd ed., Cambridge Univ. Press, London, 1950

[11] M. V. Cerrillo, Elementary introduction to the theory of saddlepoint method of integration, Research Lab. Electronics, Techn. Report no. 55:2a (1950) M.I.T., Cambridge, Mass., 1954

[12] T. C. Fang and J. M. Klosner, Expanding axial wave on a submerged cylindrical shell, Polytechnic Institute of Brooklyn, PIBAL Report no. 69-2, January 1969

[13] P. M. Morse and H. Feshbach, Methods of theoretical physics, McGraw-Hill, New York, 1961

[14] M. Abramowitz and I. A. Stegun (Editors), Handbook of mathematical functions, with formulas, graphs and mathematical tables, Nat. Bur. Standards Appl. Math. Series, 55, Superintendent of Documents, U.S. Government Printing Office, Washington, D.C., 1964; 3rd printing, with corrections, 1965

[15] R. Folk, G. Fox, C.A. Shook and C. W. Curtis, Elastic strain produced by sudden application of pressure to one end of a cylindrical bar. I: Theory, J. Acoust. Soc. Amer. 30, 552-558 (1958) 